\title{
Analytical Solutions of Spherical Cavity Expansion Near a Slope due to Pile Installation
}

\author{
Jingpei Li, ${ }^{1,2}$ Yaguo Zhang, ${ }^{1,2}$ Haibing Chen, ${ }^{1,2}$ and Fayun Liang ${ }^{1,2}$ \\ ${ }^{1}$ Key Laboratory of Geotechnical and Underground Engineering of Ministry of Education, Tongji University, Shanghai 200092, China \\ ${ }^{2}$ Department of Geotechnical Engineering, Tongji University, Shanghai 200092, China \\ Correspondence should be addressed to Jingpei Li; lijp2773@tongji.edu.cn
}

Received 5 June 2013; Accepted 18 August 2013

Academic Editor: Ga Zhang

Copyright (c) 2013 Jingpei Li et al. This is an open access article distributed under the Creative Commons Attribution License, which permits unrestricted use, distribution, and reproduction in any medium, provided the original work is properly cited.

\begin{abstract}
Based on the hypothesis that the penetration of a single pile can be simulated by a series of spherical cavity expansions, this paper presents an analytical solution of cavity expansion near the sloping ground. Compared with the cavity expansion in the half-space, the sloping free boundary has been taken into account as well as the horizontal free boundary. The sloping and horizontal free surfaces are considered by the introduction of a virtual image technique, the harmonic function, and the Boussinesq solution. The results show that the sloping free boundary and the variation of the inclination angle have pronounced influences on the distribution of the stress and displacement induced by the spherical cavity expansion. The present solution provides a simplified and realistic theoretical method to predict the soil behaviors around the spherical cavity near the sloping ground. The approach can also be used for the determination of the inclination angle of the slope according to the maximum permissible displacement.
\end{abstract}

\section{Introduction}

There are many situations where foundations need to be located on the top of a slope, such as the piled bridge abutment adjacent to a slope crest. Hence, pile installation in sloping ground has attracted wide concerns [1-3]. In contrast to the horizontal ground surface case, the boundary effects of a slope should be considered for situations of piles embedded adjacent to the slope. The existing boundary not only affects the bearing capacity of piles, but also adds the risk of slope failure [4]. For instance, a riverbank dike, located along the Bailianjing River in Shanghai, was damaged by pile driving in soft clay during the construction of a newly elevated dike [5].

Following the early suggestion $[6,7]$, solutions of the limit pressures of spherical and cylindrical cavities are used to predict the end bearing and shaft capacities of piles $[8,9]$, as well as the stress fields and lateral displacements of the surrounding subsoil induced by installation of a pile [10-13]. However, the solutions of a cavity expansion in an infinite medium do not satisfy the stress conditions at the free surface during the pile installation. Sagaseta et al. [14] and Sagaseta [15] considered the problem as strain controlled and obtained strains by using only the incompressibility condition. The presence of the top free surface was considered by means of a virtual image technique and some results for the elastic half-space. Besides, Keer et al. [16] derived a solution for the expansion of spherical cavity in a half-space by using the image source method [17] and the concept of cavity expansion source [18]. These methods can be well used to analyze the boundary effects of the free surface of the half-space, but they are not directly applicable to cavity expansion near a slope. Compared with cavity expansion in a semi-infinite half-space, the slope surface should be taken into account.

In this paper, the expansion caused by pile tip is simulated as a spherical cavity expansion. Theoretical solutions for the expansion of a single spherical cavity near slope are derived by using the virtual image approach. Meanwhile, the correction stress functions and the Boussinesq solutions are introduced to consider the effects of both horizontal ground surface and slope surface in this analysis.

\section{Basic Theories and Geometry of the Problem}

The concept of the cavity expansion source was first used by Hopkins [18]. The model shown in Figure 1 is a cavity under 


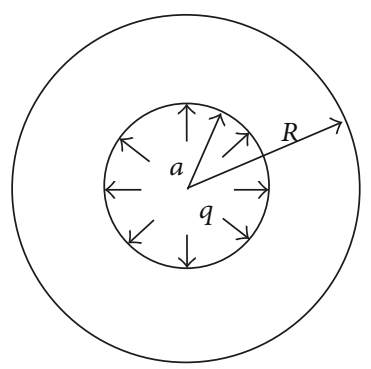

FIGURE 1: Cavity expansion source induced by the uniform pressure on the internal spherical surface.

a uniform pressure $q$ on the internal spherical surface with radius $a$.

The fields of stress and displacement induced by the pressure are as follows:

$$
\begin{gathered}
\sigma_{R}=\frac{a^{3} q}{R^{3}}, \\
\sigma_{\varphi}=\sigma_{\theta}=-\frac{a^{3} q}{2 R^{3}}, \\
u_{R}=\frac{a^{3} q}{4 G R^{2}},
\end{gathered}
$$

where the $R$ is the distance between the center and the calculation point, the $G$ is the shear modulus of the soil, and the $\sigma_{R}, \sigma_{\varphi}$, and $\sigma_{\theta}$ refer to radial, hoop, and tangential stresses, respectively. Due to the spherical symmetry, the shear stresses are equal to zero, that is, $\tau_{R \varphi}=\tau_{R \theta}=\tau_{\varphi \theta}$.

The expansion caused by the pile tip is usually simulated as a spherical cavity expansion $[19,20]$. Hence, attention will be paid to the solutions of a single cavity expansion adjacent to a slope. As shown in Figure 2, the depth of the cavity below the horizontal ground surface is denoted by $h$, and the angle between the slope and vertical direction is denoted by $\beta$. Unlike cavity expansion in an infinite medium, the analytical solutions of cavity problem near a slope are currently only possible in elastic materials. Accordingly, the soil is assumed to be an isotropic, homogeneous, and linear elastic material, and only small strains occur during the process of the cavity expansion. For simplicity, the gravitational stresses are ignored.

\section{The Solution Method}

3.1. The Expansion of a Spherical Cavity and Its Image in the Half-Space. The theoretical solution for the expansion of a single spherical cavity in a half-space has been presented by Keer et al. [16], where the free surface of the half-space is horizontal $(z=0)$. Similarly, the solutions can also be derived when the free surface is vertical $(r=0)$. Taking the vertical surface as the plane of symmetry, another virtual spherical cavity is put at the image point which is shown in Figure 3. The coordinate of the calculation point $p$ is $(r, z)$, and $t$ is the horizontal distance between the center of spherical cavity and the vertical free surface. $R_{1}$ is the distance from the spherical

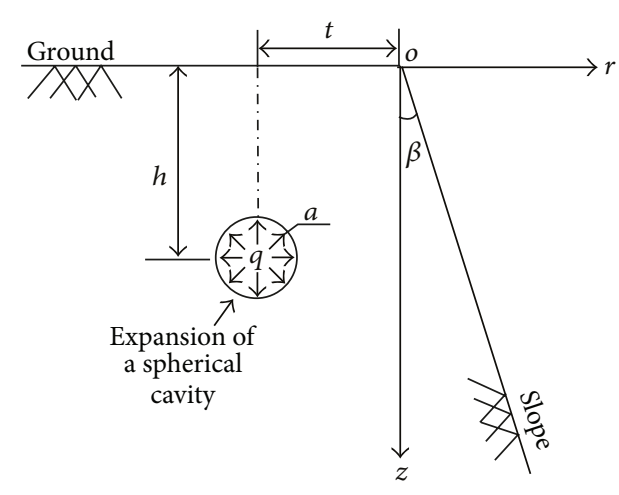

FIGURE 2: Expansion of a spherical cavity near the sloping ground.

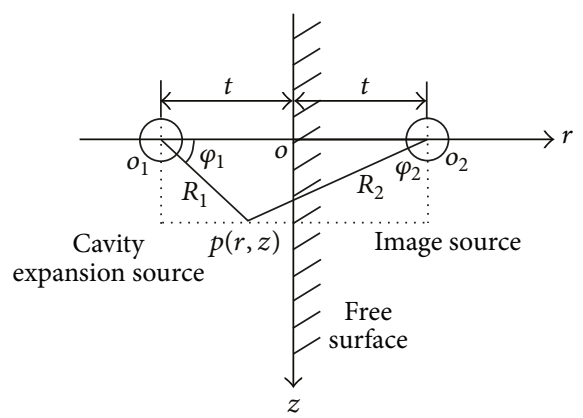

FIGURE 3: A cavity expansion source and its image.

cavity to point $p$, and $R_{2}$ is the distance from the image to point $p . \varphi_{1}$ and $\varphi_{2}$ are the angles from $r$ direction to $R_{1}$ and $R_{2}$, respectively.

The stress and displacement components of the cavity expansion in a cylindrical coordinate system can be written as

$$
\begin{gathered}
\sigma_{r}=\sigma_{R} \cos ^{2} \phi+\sigma_{\phi} \sin ^{2} \phi-\tau_{R \phi} \sin 2 \phi, \\
\sigma_{z}=\sigma_{R} \sin ^{2} \phi+\sigma_{\phi} \cos ^{2} \phi+\tau_{R \phi} \sin 2 \phi, \\
\tau_{r z}=\left(\sigma_{R}-\sigma_{\phi}\right) \sin \phi \cos \phi+\tau_{R \phi} \cos 2 \phi, \\
u_{r}=u_{R} \cos \phi, \quad u_{z}=u_{R} \sin \phi .
\end{gathered}
$$

By substituting (1) to (3) into (4) to (7) and using the principle of superposition, the stress and displacement of the spherical cavity and its image in the cylindrical coordinate are as follows:

$$
\begin{aligned}
& \sigma_{r}=\frac{a^{3} q}{2}\left[\frac{3(t+r)^{2}}{R_{1}^{5}}-\frac{1}{R_{1}^{3}}+\frac{3(r-t)^{2}}{R_{2}^{5}}-\frac{1}{R_{2}^{3}}\right], \\
& \sigma_{z}=\frac{a^{3} q}{2}\left[\frac{2}{R_{1}^{3}}-\frac{3(t+r)^{2}}{R_{1}^{5}}+\frac{2}{R_{2}^{3}}-\frac{3(r-t)^{2}}{R_{2}^{5}}\right],
\end{aligned}
$$




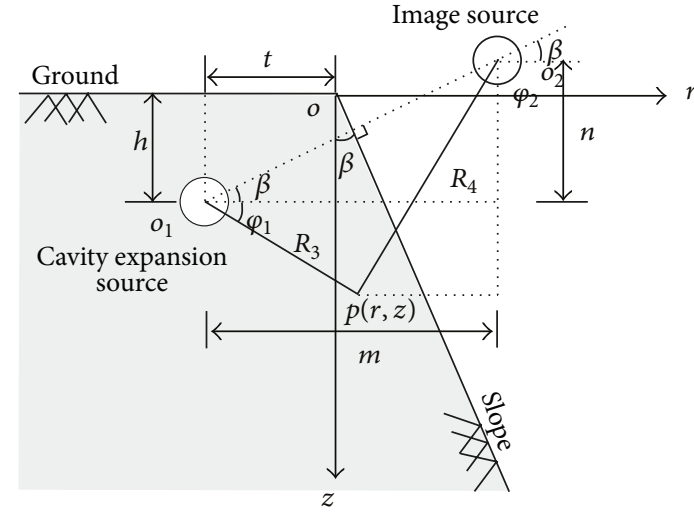

(a)

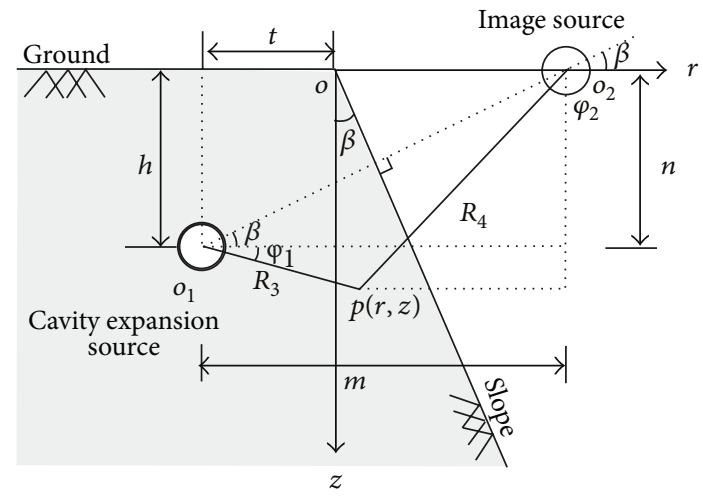

(b)

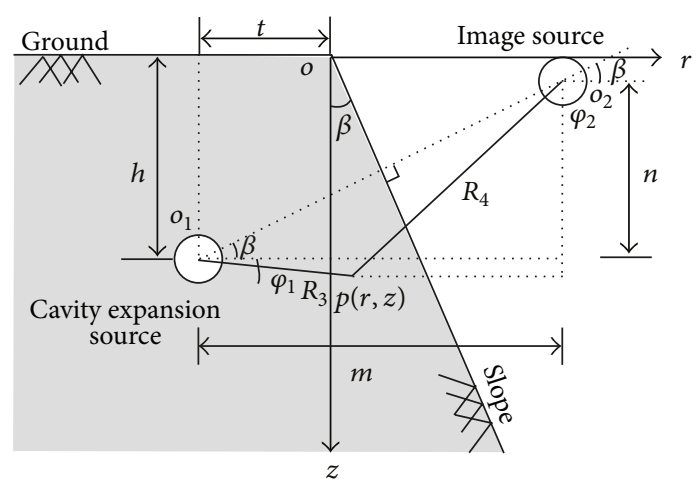

(c)

FIGURE 4: The expansion of the single spherical cavity near a slope. (a) The image source is located above the ground surface. (b) The image source is located on the ground surface. (c) The image source is located below the ground surface.

$$
\begin{gathered}
\tau_{r z}=\frac{3 a^{3} q z(t+r)}{2 R_{1}^{5}}+\frac{3 a^{3} q z(r-t)}{2 R_{2}^{5}}, \\
\sigma_{\theta}=-\frac{a^{3} q}{2 R_{1}^{3}}-\frac{a^{3} q}{2 R_{2}^{3}} \\
u_{r}=\frac{a^{3} q}{4 G}\left(\frac{t+r}{R_{1}^{3}}+\frac{r-t}{R_{2}^{3}}\right) \\
u_{z}=\frac{a^{3} q}{4 G}\left(\frac{z}{R_{1}^{3}}+\frac{z}{R_{2}^{3}}\right)
\end{gathered}
$$

where $R_{1}=\sqrt{(t+r)^{2}+z^{2}}$ and $R_{2}=\sqrt{(t-r)^{2}+z^{2}}$.

The horizontal ground and the slope are both free surfaces, on which the normal and shear stresses are zero. Because of the symmetry, the source and its image produce zero shear stress $\left(\tau_{r z}=0\right)$ and a nonzero normal stress $\left(\sigma_{r} \neq 0\right)$ on the free surface $r=0$. Thus, the presence of the normal stress violates the free surface boundary condition

$$
\left.\sigma_{r}\right|_{r=0}=a^{3} q\left(\frac{3 t^{2}}{R_{0}^{5}}-\frac{1}{R_{0}^{3}}\right),
$$

where $R_{0}=\sqrt{t^{2}+z^{2}}$.
3.2. The Expansion of a Spherical Cavity and Its Image Near a Slope. The boundary effects of both ground surface and slope should be investigated during the analysis of the cavity expansion near the sloping ground. The problem turns to be more difficult due to the increase of the slope boundary. Similarly, the virtual image technique is employed to consider the boundary effects of the slope. Taking the slope as the plane of symmetry, the image source is set at the image point as shown in Figure 4.

The solutions of the two cavities expansion (the actual spherical cavity and its virtual image) can be obtained by the principle of superposition. As a result, the stress and displacement components in the cylindrical coordinate system are shown to be

$$
\begin{aligned}
& \sigma_{r}^{(0)}=\frac{a^{3} q}{2}\left[\frac{3(t+r)^{2}}{R_{3}^{5}}-\frac{1}{R_{3}^{3}}+\frac{3(t+r-m)^{2}}{R_{4}^{5}}-\frac{1}{R_{4}^{3}}\right], \\
& \sigma_{z}^{(0)}=\frac{a^{3} q}{2}\left[\frac{2}{R_{3}^{3}}-\frac{3(t+r)^{2}}{R_{3}^{5}}+\frac{2}{R_{4}^{3}}-\frac{3(t+r-m)^{2}}{R_{4}^{5}}\right], \\
& \tau_{r z}^{(0)}=\frac{3 a^{3} q(z-h)(t+r)}{2 R_{3}^{5}}+\frac{3 a^{3} q(z-h+n)(t+r-m)}{2 R_{4}^{5}},
\end{aligned}
$$




$$
\begin{gathered}
\sigma_{\theta}^{(0)}=-\frac{a^{3} q}{2 R_{3}^{3}}-\frac{a^{3} q}{2 R_{4}^{3},} \\
u_{r}^{(0)}=\frac{a^{3} q}{4 G}\left(\frac{t+r}{R_{3}^{3}}+\frac{t+r-m}{R_{4}^{3}}\right), \\
u_{z}^{(0)}=\frac{a^{3} q}{4 G}\left(\frac{z-h}{R_{3}^{3}}+\frac{z-h+n}{R_{4}^{3}}\right),
\end{gathered}
$$

where the cavity depth below the ground surface is denoted by $h$, the distance from the source $o_{1}$ to the calculation point $p$ is $R_{3}$, and the image $o_{2}$ to the point $p$ is $R_{4}$. According to the relationship of geometry shown in Figure 4, the expressions for $R_{3}$ and $R_{4}$ are

$$
\begin{gathered}
R_{3}=\sqrt{(t+r)^{2}+(z-h)^{2}}, \\
R_{4}=\sqrt{(m-t-r)^{2}+(n+z-h)^{2}} .
\end{gathered}
$$

With the increasing of depth of the spherical cavity, the image cavity gradually moves from above ground (Figure 4(a)) to the ground below (Figure 4(c)). Accordingly, the expressions of $R_{3}$ and $R_{4}$ will be changed with the depth of cavities. Specifically, when the virtual image cavity is just on the ground surface as shown in Figure 4(b), the $R_{4}$ can be simplified to be

$$
\begin{aligned}
& R_{4}=\sqrt{(m-t-r)^{2}+z^{2}}, \\
& m=2(h \tan \beta+t) \cos ^{2} \beta, \\
& n=(h \tan \beta+t) \sin 2 \beta,
\end{aligned}
$$

where $m$ and $n$ are defined as the horizontal ( $r$ direction) and vertical ( $z$ direction) distances between the source and its image, respectively.

\subsection{The Correction of the Stresses on the Horizontal Ground.} According to the models shown in Figure 4, the actual expansion cavity and its image do produce not only nonzero normal stress but also shear stress on the horizontal ground surface $z=0$, which can be shown to be as follows:

$$
\begin{gathered}
\left.\sigma_{z}^{(0)}\right|_{z=0}=\frac{a^{3} q}{2}\left[\frac{3 h^{2}}{R_{3}^{\prime 5}}-\frac{1}{R_{3}^{\prime 3}}+\frac{3(n-h)^{2}}{R_{4}^{\prime 5}}-\frac{1}{R_{4}^{\prime 3}}\right], \\
\left.\tau_{r z}^{(0)}\right|_{z=0}=\frac{-3 a^{3} q h(t+r)}{2 R_{3}^{\prime 5}}-\frac{3 a^{3} q(h-n)(t+r-m)}{2 R_{4}^{\prime 5}},
\end{gathered}
$$

where $R_{3}^{\prime}=\sqrt{(t+r)^{2}+h^{2}}$ and $R_{4}^{\prime}=$ $\sqrt{(m-t-r)^{2}+(n-h)^{2}}$.

In order to satisfy the condition of the free horizontal boundary $(z=0)$, the different correction functions are introduced to deal with the normal stress (see (25)) and shear stress (see (26)) on the boundary. Based on the theory of superposition, the stresses on the ground surface can be divided into two parts: (i) only normal stress on the horizontal ground surface: $\left.\sigma_{z}^{(1)}\right|_{z=0}=-\left.\sigma_{z}^{(0)}\right|_{z=0},\left.\tau_{r z}^{(1)}\right|_{z=0}=0 ;$

(ii) only shear stress on the horizontal ground surface: $\left.\sigma_{z}^{(2)}\right|_{z=0}=0,\left.\tau_{r z}^{(2)}\right|_{z=0}=-\left.\tau_{r z}^{(0)}\right|_{z=0}$.

Using the axially symmetric stress function $f(r, z)$ of Kassir and Sih [21] in the first part (i), the corresponding stress and displacement solutions are written as follows:

$$
\begin{gathered}
\sigma_{r}^{(1)}=2 G\left[(1-2 \nu) \frac{\partial^{2} f}{\partial r^{2}}-2 \nu \frac{\partial^{2} f}{\partial z^{2}}+z \frac{\partial^{3} f}{\partial r^{2} \partial z}\right], \\
\sigma_{\theta}^{(1)}=2 G\left[\frac{1}{r} \frac{\partial f}{\partial r}+2 \nu \frac{\partial^{2} f}{\partial r^{2}}+\frac{z}{r} \frac{\partial^{2} f}{\partial r \partial z}\right], \\
\sigma_{z}^{(1)}=2 G\left[-\frac{\partial^{2} f}{\partial z^{2}}+z \frac{\partial^{3} f}{\partial z^{3}}\right], \\
\tau_{r z}^{(1)}=2 G z \frac{\partial^{3} f}{\partial r \partial z^{2}}, \\
u_{r}^{(1)}=(1-2 \nu) \frac{\partial f}{\partial r}+z \frac{\partial^{2} f}{\partial r \partial z} \\
u_{z}^{(1)}=-2(1-v) \frac{\partial f}{\partial r}+z \frac{\partial^{2} f}{\partial z^{2}}
\end{gathered}
$$

in which

$$
f=A\left(\frac{1}{R_{3 t}}+\frac{1}{R_{4 t}}\right),
$$

where $A$ is a constant. The horizontal ground surface is treated as the plane of symmetry. $R_{3 t}$ denotes the distance between the point $p$ and the symmetrical position of the actual cavity $O_{1}$. Likewise, $R_{4 t}$ is the distance from the point $p$ to the symmetrical position of the image cavity $\mathrm{O}_{2}$. As a result, their expressions can be written as follows:

$$
\begin{gathered}
R_{3 t}=\sqrt{(t+r)^{2}+(z+h)^{2}}, \\
R_{4 t}=\sqrt{[m-(t+r)]^{2}+[z-(n-h)]^{2}} ;
\end{gathered}
$$

$f$ is a harmonic function, that is, $\nabla^{2} f=0$. The corresponding stresses on the free surface are:

$$
\begin{gathered}
\left.\sigma_{z}^{(1)}\right|_{z=0}=2 G A\left[\frac{1}{R_{3 t}^{\prime 3}}-\frac{3 h^{2}}{R_{3 t}^{\prime 5}}+\frac{1}{R_{4 t}^{\prime 3}}-\frac{3(n-h)^{2}}{R_{4 t}^{\prime 5}}\right], \\
\left.\tau_{r z}^{(1)}\right|_{z=0}=0,
\end{gathered}
$$

where $R_{3 t}^{\prime}=R_{3}^{\prime}$, and $R_{4 t}^{\prime}=R_{4}^{\prime}$.

Thus, the normal stress on the horizontal ground surface $z=0$ could be eliminated by the stress function $f$ with accurate value of $A$. According to the following equation:

$$
\begin{aligned}
2 G A & {\left[\frac{1}{R_{3 t}^{\prime 3}}-\frac{3 h^{2}}{R_{3 t}^{\prime 5}}+\frac{1}{R_{4 t}^{\prime 3}}-\frac{3(n-h)^{2}}{R_{4 t}^{\prime 5}}\right] } \\
& =\frac{a^{3} q}{2}\left[\frac{3 h^{2}}{R_{3}^{\prime 5}}-\frac{1}{R_{3}^{\prime 3}}+\frac{3(n-h)^{2}}{R_{4}^{\prime 5}}-\frac{1}{R_{4}^{\prime 3}}\right],
\end{aligned}
$$


it can be obtained that

$$
A=-\frac{a^{3} q}{4 G} .
$$

Substituting the above expression of $A$ back into (33), one has

$$
f=-\frac{a^{3} q}{4 G}\left(\frac{1}{R_{3 t}}+\frac{1}{R_{4 t}}\right),
$$

and then the stress and displacement components can be obtained by substituting (39) into (27) to (32).

For the second part (ii), there is only shear stress on the horizontal ground surface. In the same way, the stress harmonic function $g(r, z)$ is given to eliminate the shear on the boundary, and the solutions of the stress and displacement are as follows:

$$
\begin{gathered}
\sigma_{r}^{(2)}=2 G\left(2 \frac{\partial^{2} g}{\partial r^{2}}-\frac{2 \nu}{r} \frac{\partial g}{\partial r}+z \frac{\partial^{3} g}{\partial r^{2} \partial z}\right), \\
\sigma_{\theta}^{(2)}=2 G\left(\frac{1}{r} \frac{\partial g}{\partial r}+2 \nu \frac{\partial^{2} g}{\partial r^{2}}+\frac{z}{r} \frac{\partial^{2} g}{\partial r \partial z}\right), \\
\sigma_{z}^{(2)}=2 G z \frac{\partial^{3} g}{\partial z^{3}} \\
\tau_{r z}^{(2)}=2 G\left(\frac{\partial^{2} g}{\partial r \partial z}+z \frac{\partial^{3} g}{\partial r \partial z^{2}}\right) \\
u_{r}^{(2)}=2(1-\nu) \frac{\partial g}{\partial r}+z \frac{\partial^{2} g}{\partial r \partial z} \\
u_{z}^{(2)}=-(1-2 \nu) \frac{\partial g}{\partial r}+z \frac{\partial^{2} g}{\partial z^{2}}
\end{gathered}
$$

Similarly, the expression of stress function can be written as follows:

$$
g=B\left(\frac{1}{R_{3 t}}+\frac{1}{R_{4 t}}\right),
$$

where $B$ is a constant. The stress function $g$ satisfies the equilibrium $\nabla^{2} g=0$, and the corresponding stress components on the ground surface $z=0$ are

$$
\begin{gathered}
\left.\sigma_{z}^{(2)}\right|_{z=0}=0 \\
\left.\tau_{r z}^{(2)}\right|_{z=0}=2 G B\left[\frac{3 h(t+r)}{R_{3 t}^{\prime}}+\frac{3(h-n)(t+r-m)}{R_{4 t}^{\prime}}\right] .
\end{gathered}
$$

Accordingly, it can be obtained that $B=-a^{3} q / 4 G$. follows:

The final expression for the stress function is then as

$$
g=-\frac{a^{3} q}{4 G}\left(\frac{1}{R_{3 t}}+\frac{1}{R_{4 t}}\right) .
$$

Substituting (48) into (40) to (45), the stress and displacement components can be obtained.

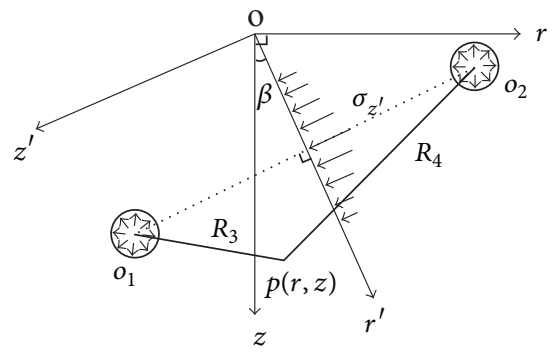

FIgURE 5: The stress distribution of the slope surface.

3.4. The Correction of the Stresses on the Slope Surface. Considering a virtual source, positive mirror image of the actual cavity with respect to a slope surface will produce the same normal stresses and opposite shear stresses as the actual cavity, the shear stress is eliminated, and the normal stress increases doubly, as shown in Figure 5.

Using the method of coordinate transformation, the normal and shear stresses can be obtained as follows:

$$
\begin{aligned}
&\left.\sigma_{z^{\prime}}\right|_{z^{\prime}=0}= 3 a^{3} q\left[\frac{\left(t+r^{\prime} \sin \beta\right)^{2}+c^{2}}{\left.R_{0}^{5}-\frac{1}{R_{0}^{3}}\right]}\right. \\
& \times \cos ^{2} \beta-3 a^{3} q \cdot\left[\frac{\left(r^{\prime} \cos \beta-h\right)\left(t+r^{\prime} \sin \beta\right)+c d}{2 R_{0}^{5}}\right] \\
& \times \sin 2 \beta+\frac{a^{3} q}{2}\left[\frac{4}{R_{0}^{3}}-\frac{3\left(t+r^{\prime} \sin \beta\right)^{2}+3 c^{2}}{R_{0}^{5}}\right] \\
&\left.\tau_{r^{\prime} z^{\prime}}\right|_{z^{\prime}=0}=0,
\end{aligned}
$$

where $c=t+r^{\prime} \sin \beta-m, d=r^{\prime} \cos \beta-h+n$, and $R_{0}=$ $\sqrt{\left(t+r^{\prime} \sin \beta\right)^{2}+\left(r^{\prime} \cos \beta-h\right)^{2}}$.

In order to satisfy the free surface boundary condition as much as possible, the Boussinesq solution has been introduced to correct the normal stress. Stress $q^{\prime}$ is applied on the surface of the slope, which is equal to the normal stress $\left.\sigma_{z^{\prime}}\right|_{z^{\prime}=0}$ in value but opposite in direction.

As shown in Figure 6, ol is the intersection line of the slope and the horizontal plane. A small element with an area of $\rho d \theta d \rho$ is taken out of the slope lor' (i.e., or ${ }^{\prime}$ in Figure 5) for analysis. Further, the force exerted on the small element is equal to $q^{\prime} \rho d \theta d \rho$. Using the Boussinesq solutions, the stress and displacement components of soil under the action of the force $\left(q^{\prime} \rho d \theta d \rho\right)$ can be derived as follows:

$$
\begin{gathered}
d \sigma_{z^{\prime}}=\frac{3 q^{\prime} z^{\prime 3}}{2 \pi R^{\prime 5}} \rho d \theta d \rho, \\
d \sigma_{\theta^{\prime \prime}}=\frac{(1-2 \nu) q^{\prime}}{2 \pi R^{\prime 2}}\left(\frac{R^{\prime}}{R^{\prime}+z^{\prime}}-\frac{z^{\prime}}{R^{\prime}}\right) \rho d \theta d \rho, \\
d \sigma_{r^{\prime \prime}}=\frac{q^{\prime}}{2 \pi R^{\prime 2}}\left[\frac{3 r^{\prime \prime 2} z^{\prime}}{R^{\prime 3}}-\frac{(1-2 \nu) R^{\prime}}{R^{\prime}+z^{\prime}}\right] \rho d \theta d \rho,
\end{gathered}
$$




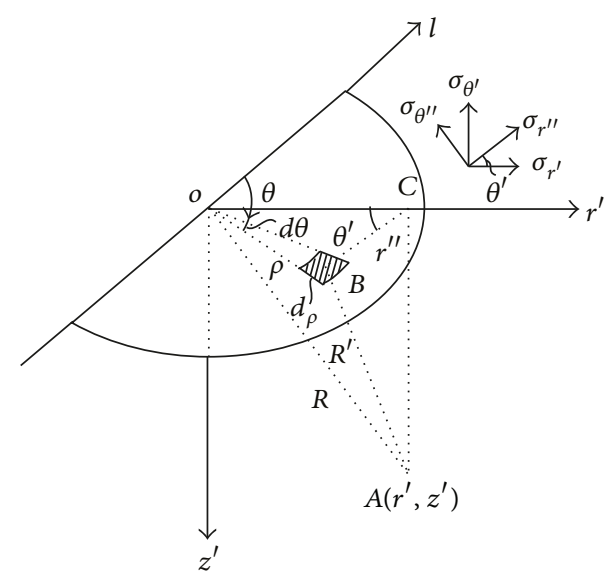

Figure 6: The stress analysis of calculation point by the force on the small element.

$$
\begin{gathered}
d \tau_{r^{\prime \prime} z^{\prime}}=\frac{3 q^{\prime} r^{\prime \prime} z^{\prime 2}}{2 \pi R^{\prime 5}} \rho d \theta d \rho \\
d u_{z^{\prime}}=\frac{(1+\nu) q^{\prime}}{2 \pi E R^{\prime}}\left[2(1-v)+\frac{z^{\prime 2}}{R^{\prime 2}}\right] \rho d \theta d \rho \\
d u_{r^{\prime \prime}}=\frac{(1+\nu) q^{\prime}}{2 \pi E R^{\prime}}\left[\frac{r^{\prime \prime} z^{\prime}}{R^{\prime 2}}-\frac{(1-2 v) r^{\prime \prime}}{R^{\prime}+z^{\prime}}\right] \rho d \theta d \rho
\end{gathered}
$$

in which

$$
\begin{gathered}
r^{\prime \prime 2}=\rho^{2}+r^{\prime 2}-2 \rho r^{\prime} \cos \left(\theta-\frac{\pi}{2}\right), \\
R^{\prime 2}=r^{\prime \prime 2}+z^{\prime 2}=\rho^{2}+r^{\prime 2}-2 \rho r^{\prime} \cos \left(\theta-\frac{\pi}{2}\right)+z^{\prime 2} .
\end{gathered}
$$

The directions of stress and displacement induced by the force on the small element are not always in accordance with coordinate axes (Figure 6). By means of coordinate transformation, the solutions of stresses and displacements in the coordinate system $r^{\prime} o z^{\prime}$ (Figure 5) can be obtained. After that, integrating results with respect to the whole sloping ground surface can lead to the following:

$$
\begin{gathered}
\sigma_{z^{\prime}}=\int_{0}^{\pi} \int_{0}^{\infty} d \sigma_{z^{\prime}}, \\
\sigma_{\theta^{\prime}}=\int_{0}^{\pi} \int_{0}^{\infty}\left(d \sigma_{r^{\prime \prime}} \sin ^{2} \theta^{\prime}+d \sigma_{\theta^{\prime \prime}} \cos ^{2} \theta^{\prime}\right), \\
\sigma_{r^{\prime}}=\int_{0}^{\pi} \int_{0}^{\infty}\left(d \sigma_{r^{\prime \prime}} \cos ^{2} \theta^{\prime}+d \sigma_{\theta^{\prime \prime}} \sin ^{2} \theta^{\prime}\right), \\
\tau_{r^{\prime} z^{\prime}}=\int_{0}^{\pi} \int_{0}^{\infty} d \tau_{r^{\prime \prime} z^{\prime}} \cos \theta^{\prime}, \\
u_{z^{\prime}}=\int_{0}^{\pi} \int_{0}^{\infty} d u_{z^{\prime}}, \\
u_{r^{\prime}}=\int_{0}^{\pi} \int_{0}^{\infty} d u_{r^{\prime \prime}} \cos \theta^{\prime} .
\end{gathered}
$$

Hence, the stress and displacement components in the coordinate system roz can be derived as follows:

$$
\begin{gathered}
\sigma_{r}^{(3)}=\sigma_{r^{\prime}} \sin ^{2} \beta+\sigma_{z^{\prime}} \cos ^{2} \beta-\tau_{r^{\prime} z^{\prime}} \sin 2 \beta, \\
\sigma_{z}^{(3)}=\sigma_{r^{\prime}} \cos ^{2} \beta+\sigma_{z^{\prime}} \sin ^{2} \beta+\tau_{r^{\prime} z^{\prime}} \sin 2 \beta, \\
\tau_{r z}^{(3)}=\frac{1}{2}\left(\sigma_{r^{\prime}}-\sigma_{z^{\prime}}\right) \sin 2 \beta-\tau_{r^{\prime} z^{\prime}} \cos 2 \beta, \\
\sigma_{\theta}^{(3)}=\sigma_{\theta^{\prime}}, \\
u_{r}^{(3)}=u_{r^{\prime}} \sin \beta-u_{z^{\prime}} \cos \beta, \\
u_{z}^{(3)}=u_{r^{\prime}} \cos \beta+u_{z^{\prime}} \sin \beta,
\end{gathered}
$$

where $\theta^{\prime}=\arctan \left(\rho \sin (\theta-(\pi / 2)) /\left(r^{\prime}-\rho \cos (\theta-(\pi / 2))\right)\right)$, $r^{\prime}=r \sin \beta+z \cos \beta$, and $z^{\prime}=-r \cos \beta+z \sin \beta$.

With (53), the stress and displacement fields induced by the stresses $q^{\prime}$ on the slope can be derived. Using a virtual source of the actual cavity at the image point, the lateral deformations of soil around spherical cavity were predicted by Rao et al. [22]. Meanwhile, the Cerruti solutions are used to eliminate the shear stresses produced by the expansion of both the real and the imaginary spherical cavities in an infinite space. However, their results are inappropriate because of the incorrect using of the Cerruti solutions. It is known that a distributed stress is clearly not a point force in the elementary sense. Hence, the stress should be integrated with respect to the slope surface when it is substituted into the Cerruti solutions.

Accordingly, when considering the effects of both horizontal and sloping free boundaries, the final results of the expansion of a single spherical cavity near a slope (Figure 2) can be obtained by superposition of all the parts stresses and displacements:

$$
\begin{aligned}
& \sigma_{r}=\sigma_{r}^{(0)}+\sigma_{r}^{(1)}+\sigma_{r}^{(2)}+\sigma_{r}^{(3)}, \\
& \sigma_{z}=\sigma_{z}^{(0)}+\sigma_{z}^{(1)}+\sigma_{z}^{(2)}+\sigma_{z}^{(3)}, \\
& \sigma_{\theta}=\sigma_{\theta}^{(0)}+\sigma_{\theta}^{(1)}+\sigma_{\theta}^{(2)}+\sigma_{\theta}^{(3)}, \\
& \tau_{r z}=\tau_{r z}^{(0)}+\tau_{r z}^{(1)}+\tau_{r z}^{(2)}+\tau_{r z}^{(3)}, \\
& u_{r}=u_{r}^{(0)}+u_{r}^{(1)}+u_{r}^{(2)}+u_{r}^{(3)}, \\
& u_{z}=u_{z}^{(0)}+u_{z}^{(1)}+u_{z}^{(2)}+u_{z}^{(3)} .
\end{aligned}
$$

\section{Discussion of the Solutions}

The presence of the horizontal and sloping free surfaces is considered in this paper. Consequently, the present solutions have more extensive applications compared with solutions of Keer et al. [16]. According to Section 3, the solutions for this problem can be derived in four steps (cavity expansion in an infinite medium, cavity and its image expansion in an infinite medium, and the corrections of stresses on horizontal surface 
and sloping surface). The range of the angle $\beta$ between the slope and the vertical plane is $0 \leqslant \beta \leqslant \pi / 2$.

When $\beta=0$, the slope turns to be vertical plane, as shown in Figure 3. Meanwhile, it can be derived from (23) and (24) that the horizontal distance between the actual cavity and its image is $m=2 t$, while the vertical distance is $n=0$. As a result, (15) to (20) convert into (8) to (13) in sequence.

When $\beta=\pi / 2$, there is only a free surface in the horizontal direction. In this case, substituting $\beta$ into (23) and (24), $m=0$ and $n=2 h$ can be obtained. Accordingly, (15) converts into equations that were proposed by Keer et al. [16].

When $0<\beta<\pi / 2$, there are horizontal and sloping free boundaries as described in this paper. Therefore, the solutions of expansion of spherical cavity in half-space with horizontal and vertical free boundaries or only a horizontal free boundary are the particular cases of present solutions.

As stated above, (15) to (20) can degenerate to the existing solutions for the extreme cases of horizontal ground and vertical slope, which demonstrates the correctness of solutions derived by the first two steps. Steps 3 and 4 involve the correction of stresses on free surface, which is based on an understanding that existing stresses can be offset by the stresses with the same magnitude and opposite direction. In fact, effects of Steps 3 and 4 are further demonstrated by the analysis cases below.

\section{Results and Parameters Analysis}

In order to consider all the stress components together, the variation of the Mises stress during the expansion of the cavity is analyzed. The parameters in the example analyses are $q=200 \mathrm{kPa}, E=5000 \mathrm{kPa}, a=0.25 \mathrm{~m}$, and $v=0.5$ (incompressible undrained clay). As the distance $(t+r) / a$ increases or the sloping free boundary is approached, the Mises stress decreases very rapidly in the range $2 \leqslant(t+$ $r) / a \leqslant 3$, but then it decreases it decreases more slowly with further increase of the distance $(t+r) / a$, as shown in Figure 7. In order to give a further discussion of the influence from the inclination angle of the slope, the analysis covers with different angles: $\beta=15^{\circ}, \beta=30^{\circ}, \beta=45^{\circ}, \beta=60^{\circ}$, and $\beta=75^{\circ}$. The corresponding Mises stresses are shown in Figure 7, respectively. Evidently, the angle $\beta$ has a pronounced influence on the distributions of the Mises stress. At the same point near the spherical cavity, the Mises stress increases with the increase of the angle $\beta$. For example, the Mises stress rises from $5.6 \times 10^{-4} \mathrm{kPa}$ to $1.3 \times 10^{-3} \mathrm{kPa}$, again at the instant of $(t+r) / a=4$, while the $\beta$ increases from $15^{\circ}$ to $75^{\circ}$. This is consistent with the results for spherical cavity expansion in half-space reported by Keer et al. [16]. The function $f_{M}$ in Figure 7 represented the Mises stress: $f_{M}=k_{2} /\left(E / h^{3}\right)$. Here, $k_{2}=\sqrt{I_{2}}\left(I_{2}\right.$ is the second principle invariance).

If the free surfaces are not taken into account, normal and shear stresses will appear at the place where there ought to be the free surface, so violating the imposed boundary condition of a free surface $[13,15,16,23]$. As described in Section 3.4, the shear stress on the sloping ground can be eliminated by using the virtual image technique. In order to cancel the normal stress on sloping surface as much as possible, the Boussinesq solutions are introduced. Another group of normal and shear

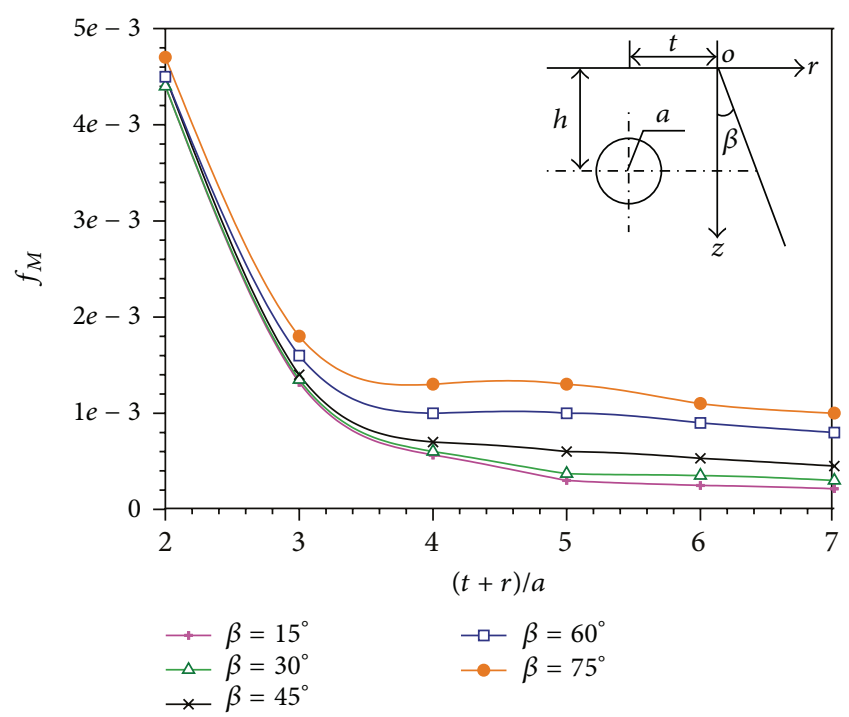

FIgURE 7: The Mises stress varies with the distance $(t+r) / a$.

stresses is produced again when the Boussinesq solutions are applied to correct stresses on the sloping surface, although the original stresses on horizontal free surface have been removed by introduction of harmonic functions (see (39) and (48)). That is because the Boussinesq solutions are aimed at half-space problems.

Figures 8(a) and 8(b) show the normal and shear stresses that vary along the horizontal free surface, respectively, where $D$ is the distance from origin of the coordinate on the horizontal free surface to the opposite direction of the $r$-axis. The stresses induced by the cavity expansion in an infinite space show large variations, which are determined by the distance from the center to the calculation point. Compared with solutions in an infinite space, both normal and shear stresses on the horizontal free surface corrected by the introduction of the harmonic functions are in close proximity to zero (Figure 8), which demonstrates the validity of the present method. For example, the maximum of the normal stress calculated by present method falls from $-0.95 \mathrm{kPa}$ to $-0.029 \mathrm{kPa}$, and the shear stress falls from $0.14 \mathrm{kPa}$ to $0.047 \mathrm{kPa}$ in case that $h=7 a$. With the increase of the distance $D / a$, the normal and shear stresses on the horizontal free surface decrease gradually. This is because the influence from the stresses on sloping surface weakens when the distance $D / a$ increases.

For the sloping free surface, although stresses have been offset by means of virtual image technique, both normal and shear stresses on the sloping surface are produced again when the harmonic functions (see (39) and (48)) are used to correct the stresses on horizontal free surface. Figures 9 (a) and 9 (b) show, respectively, the variations of the normal and shear stresses vary along the sloping free surface in cases $l=4 a$ and $5 a$, where $D$ is the distance from origin of the coordinate to an arbitrary point on the sloping free surface and $l$ is the distance between the slope and the center of the spherical cavity. Compared to solutions in an infinite space, the stresses on the sloping free surface have been partly corrected by 


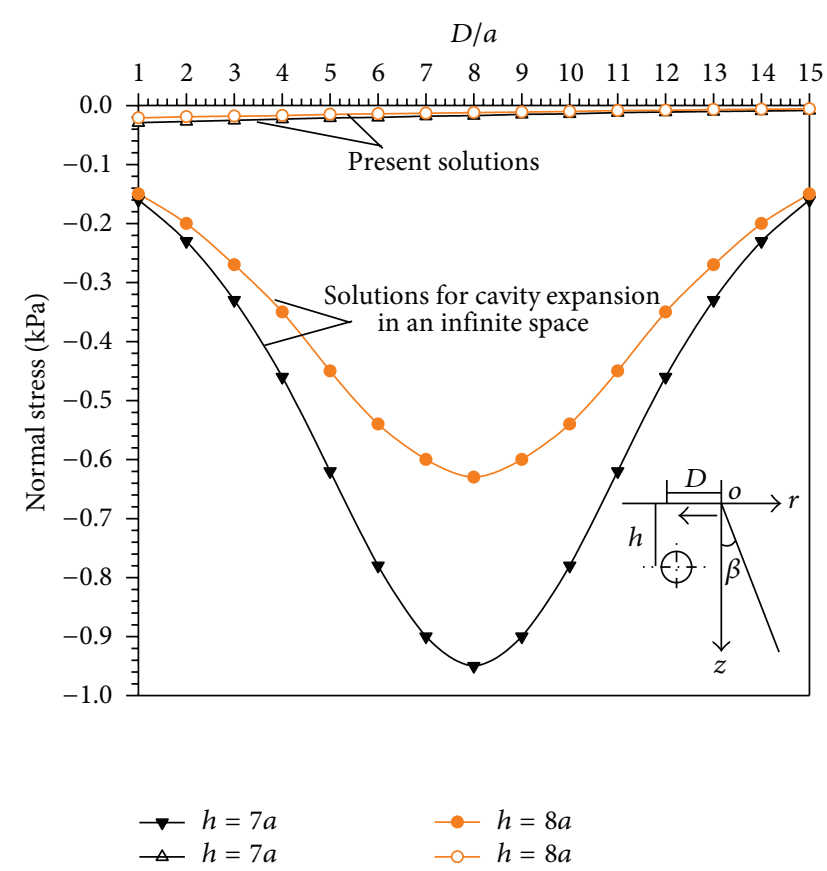

(a) Normal stress

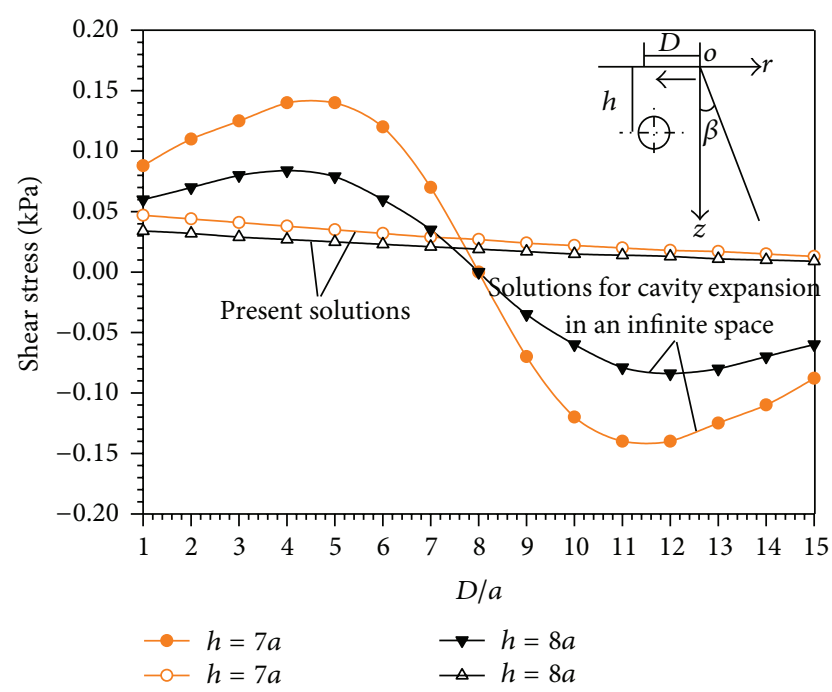

(b) Shear stress

FIGURE 8: Stress along the horizontal free surface.

the introduction of the correction functions. Figures 9(a) and 9 (b) show that, after being corrected, both the normal and shear stresses still have larger values in the range $1 \leqslant$ $D / a \leqslant 4$ and then decrease with further increase of $D / a$. The larger distance of $D / a$, the smaller the stresses on the slope surface will be produced. The condition of zero stress at the horizontal and sloping free surface is not strictly satisfied. However, this approximate approach is purposeful in reality.

From Figures 8 and 9, the stresses, particularly the shear stresses, on the two free surfaces are close to zero after correction. Thus, the stresses will decrease until zero if the correction processes are iterated continually. It is obvious that the stresses on the free surface decline slowly with the

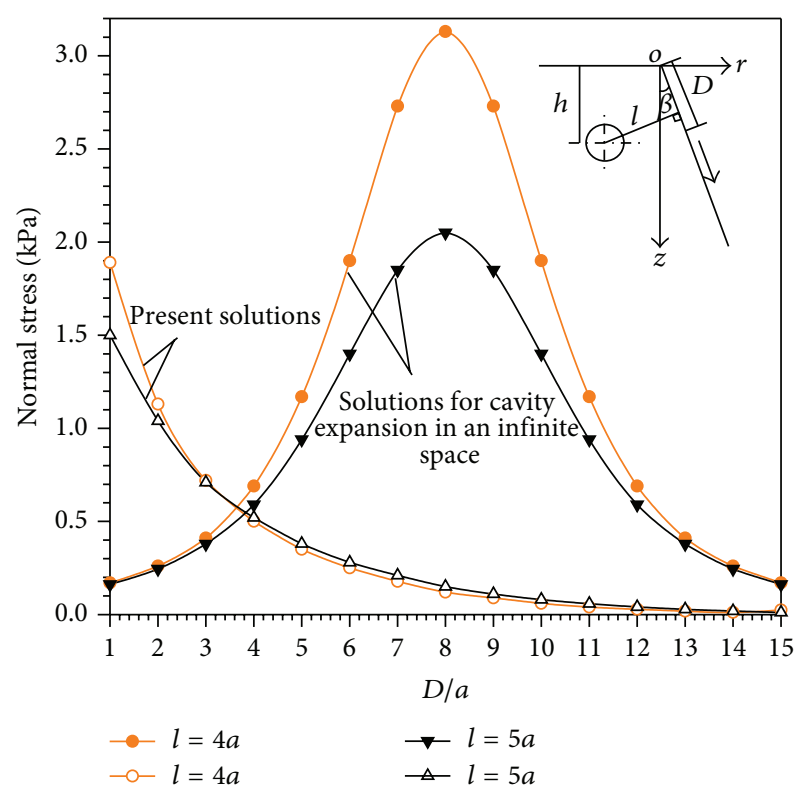

(a) Normal stress

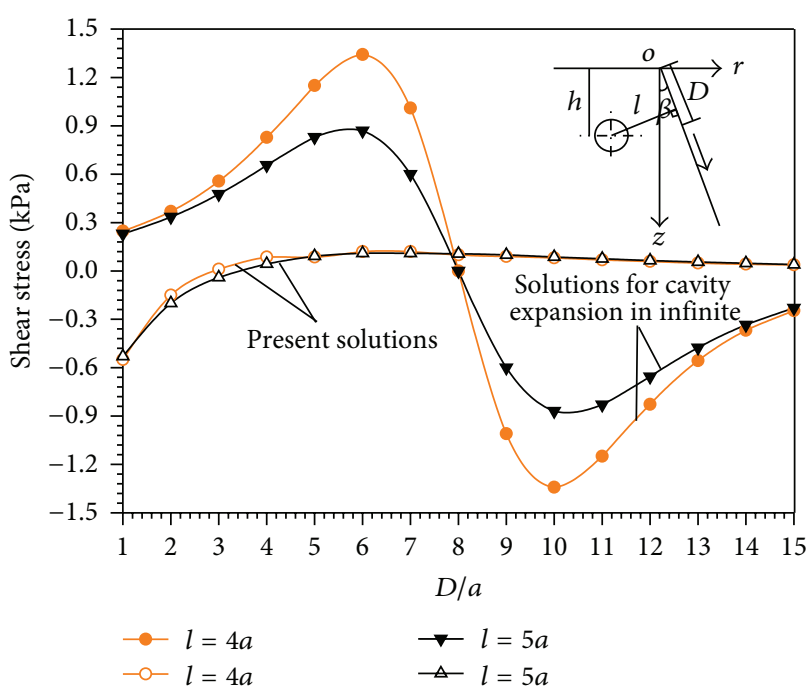

(b) Shear stress

FiguRE 9: Stress along the sloping free surface.

iteration increase, so further trivial corrections are not carried out here.

The displacement of soil induced by the cavity expansion is discussed in this section. Figure 10 illustrates that the slope and its inclination angles have pronounced influence on the distributions of the displacements induced by a cavity expansion source. The displacement decreases with the increase of the distance $(t+r) / a$. Similarly, the displacement decreases rapidly in the range $1 \leqslant(t+r) / a \leqslant 3$, but then more slowly with further increase of $(t+r) / a$. Meanwhile, the displacement induced by a cavity expansion source approaching a free surface, in general, is larger than that in infinite medium (no boundary effect). The findings are in accordance with the results proposed by Keer et al. [16] 


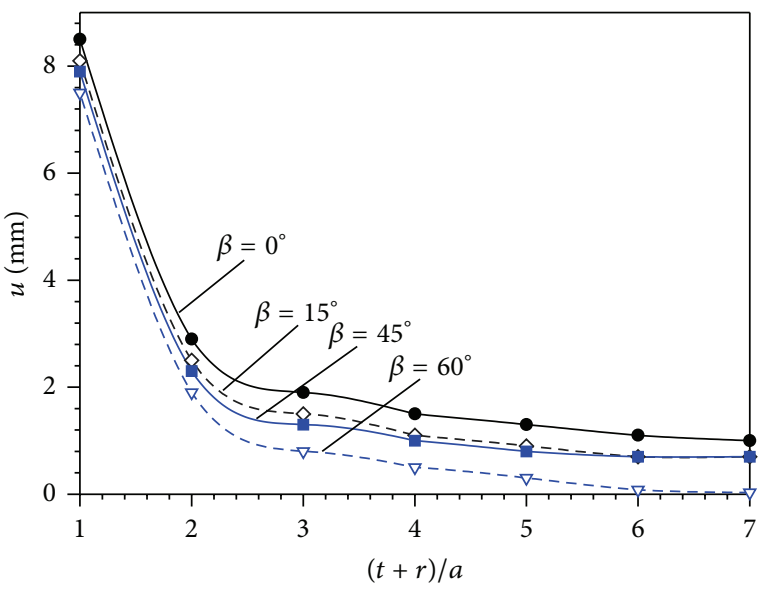

FIgURE 10: The displacement varies with distance.

and Chai et al. [20]. Here, $u=\sqrt{u_{r}^{2}+u_{z}^{2}}\left(u_{r}\right.$ and $u_{z}$ are the displacements in the $r$ and $z$ directions, resp.).

Using the theory of the spherical cavity expansion in an infinite space implies that there is an infinitely thick "soil wall" existing on the side of the slope, which will restrain the lateral displacement induced by the spherical expansion. According to the Figure 2, the slope tends to be flat with the increase of the angle $\beta$. As a result, the displacement at the same point in the soil decreases due to the increase of the thickness of "restriction." For instance, when $\beta=0^{\circ}$, the displacement is 2.4 times of the displacement induced by cavity with no boundary effect at the instant of $(t+r) / a=3$. Thus, the presence and inclination angle of the sloping free boundary have a great influence on the displacement due to cavity expansion.

\section{Conclusions}

Analytical solutions of the cavity expansion near the sloping ground were proposed based on the understanding that expansion caused by pile tip can be simulated as a spherical cavity expansion. Both the horizontal and sloping free surfaces are taken into account by using of a virtual image technique, harmonic functions, and the Boussinesq solutions, and the solutions will convert into the solutions reported by Keer et al. [16] when the sloping ground turn to the horizontal direction.

The results show that the presence and inclination angle of sloping free boundary have a considerable influence on the distributions of the stress and displacement fields induced by the spherical cavity expansion. As the distance from the cavity increases or when the boundary is approached, the Mises stress decreases. With the increase of the angle $\beta$ between the slope and the vertical plane, the slope tends to flat and the displacement at the same point in the soil decreases with the increase of the "lateral restriction." Likewise, the displacement increases with the decrease of the angle $\beta$. Therefore, the existence of the slope increases the risk of the slope failure due to the pile installation.

\section{Acknowledgment}

The work reported herein was supported by the National Natural Science Foundation of China (Grant no. 41272288). The above financial support is gratefully acknowledged.

\section{References}

[1] K. Georgiadis and M. Georgiadis, "Undrained lateral pile response in sloping ground," Journal of Geotechnical and Geoenvironmental Engineering, vol. 136, no. 11, pp. 1489-1500, 2010.

[2] C. W. W. Ng, L. M. Zhang, and K. K. S. Ho, "Influence of laterally loaded sleeved piles and pile groups on slope stability," Canadian Geotechnical Journal, vol. 38, no. 3, pp. 553-566, 2001.

[3] L. M. Zhang, C. W. W. Ng, and C. J. Lee, "Effects of slope and sleeving on the behavior of laterally loaded piles," Soils and Foundations, vol. 44, no. 4, pp. 99-108, 2004.

[4] C. Sagaseta and A. J. Whittle, "Prediction of ground movements due to pile driving in clay," Journal of Geotechnical and Geoenvironmental Engineering, vol. 127, no. 1, pp. 55-66, 2001.

[5] S. L. Shen, J. Han, H. H. Zhu, and Z. S. Hong, "Evaluation of a dike damaged by pile driving in soft clay," Journal of Performance of Constructed Facilities, vol. 19, no. 4, pp. 300-307, 2005.

[6] R. F. Bishop, R. Hill, and N. F. Mott, "The theory of indentation and hardness tests," Proceedings of the Physical Society, vol. 57, no. 3, pp. 147-159, 1945.

[7] R. Hill, The Mathematical Theory of Plasticity, Oxford University Press, Oxford, UK, 1950.

[8] H. S. Yu and G. T. Houlsby, "Finite cavity expansion in dilatant soils: loading analysis," Geotechnique, vol. 41, no. 2, pp. 173-183, 1991.

[9] M. F. Randolph, J. Dolwin, and R. Beck, "Design of driven piles in sand," Geotechnique, vol. 44, no. 3, pp. 427-448, 1994.

[10] M. F. Randolph, J. P. Carter, and C. P. Wroth, "Driven piles in clay-the effects of installation and subsequent consolidation," Geotechnique, vol. 29, no. 4, pp. 361-393, 1979.

[11] J. M. Pestana, C. E. Hunt, and J. D. Bray, "Soil deformation and excess pore pressure field around a closed-ended pile," Journal of Geotechnical and Geoenvironmental Engineering, vol. 128, no. 1, pp. 1-12, 2002.

[12] S. L. Chen and N. Y. Abousleiman, "Exact undrained elastoplastic solution for cylindrical cavity expansion in modified Cam Clay soil,” Geotechnique, vol. 62, no. 5, pp. 447-456, 2012.

[13] J. C. Chai, N. Miura, and H. Koga, "Lateral displacement of ground caused by soil-cement column installation," Journal of Geotechnical and Geoenvironmental Engineering, vol. 131, no. 5, pp. 623-632, 2005.

[14] C. Sagaseta, G. T. Houlsby, J. Norbury, and A. A. Wheeler, "Quasi-static undrained expansion of a cylindrical cavity in clay in the Presence of shaft friction and anisotropic initial stresses," Report, Department of Engineering Science, University of Oxford, 1984.

[15] C. Sagaseta, "Analysis of undrained soil deformation due to ground loss," Geotechnique, vol. 37, no. 3, pp. 301-320, 1987.

[16] L. M. Keer, Y. Xu, and V. K. Luk, "Boundary effects in penetration or perforation," Journal of Applied Mechanics, vol. 65, no. 2, pp. 489-496, 1998.

[17] R. D. Mindlin and D. H. Cheng, "Nuclei of strain in the semiinfinite solid," Journal of Applied Physics, vol. 21, pp. 926-930, 1950. 
[18] H. G. Hopkins, "Dynamic expansion of spherical cavities in metals," in Progress in Solid Mechanics, I. N. Sneddon and R. Hill, Eds., vol. 1, chapter 3, pp. 83-164, North-Holland, Amsterdam, The Netherlands, 1960.

[19] A. S. Vesic, "Expansion of cavities in infinite soil mass," Journal of the soil Mechanics and Foundations Division, vol. 98, no. 3, pp. 265-290, 1972.

[20] J. C. Chai, J. P. Carter, N. Miura, and H. H. Zhu, "Improved prediction of lateral deformations due to installation of soil-cement columns," Journal of Geotechnical and Geoenvironmental Engineering, vol. 135, no. 12, pp. 1836-1845, 2009.

[21] M. K. Kassir and G. C. Sih, Three-Dimensional Crack Problems, Noordhoff International Publishing, Dordrecht, The Netherlands, 1975.

[22] P. P. Rao, J. P. Li, and Y. Liu, "Analysis of soil squeezing effect for spherical cavity expansion with inclined nonaxisymmetric displacement boundary," Rock and Soil Mechanics, vol. 32, no. 9, pp. 2681-2687, 2011 (Chinese).

[23] H. S. Yu, Cavity Expansion Method in Geomechanics, Kluwer Academic Publishers, Dordrecht, The Netherlands, 2000. 


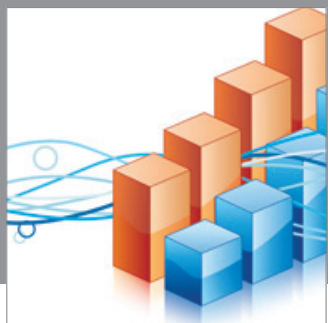

Advances in

Operations Research

mansans

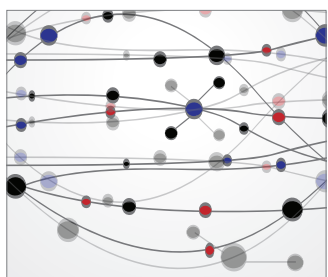

The Scientific World Journal
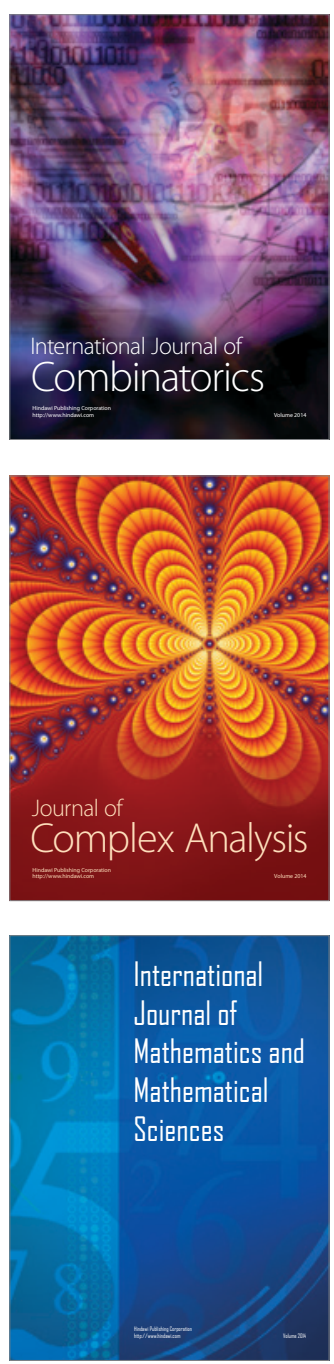
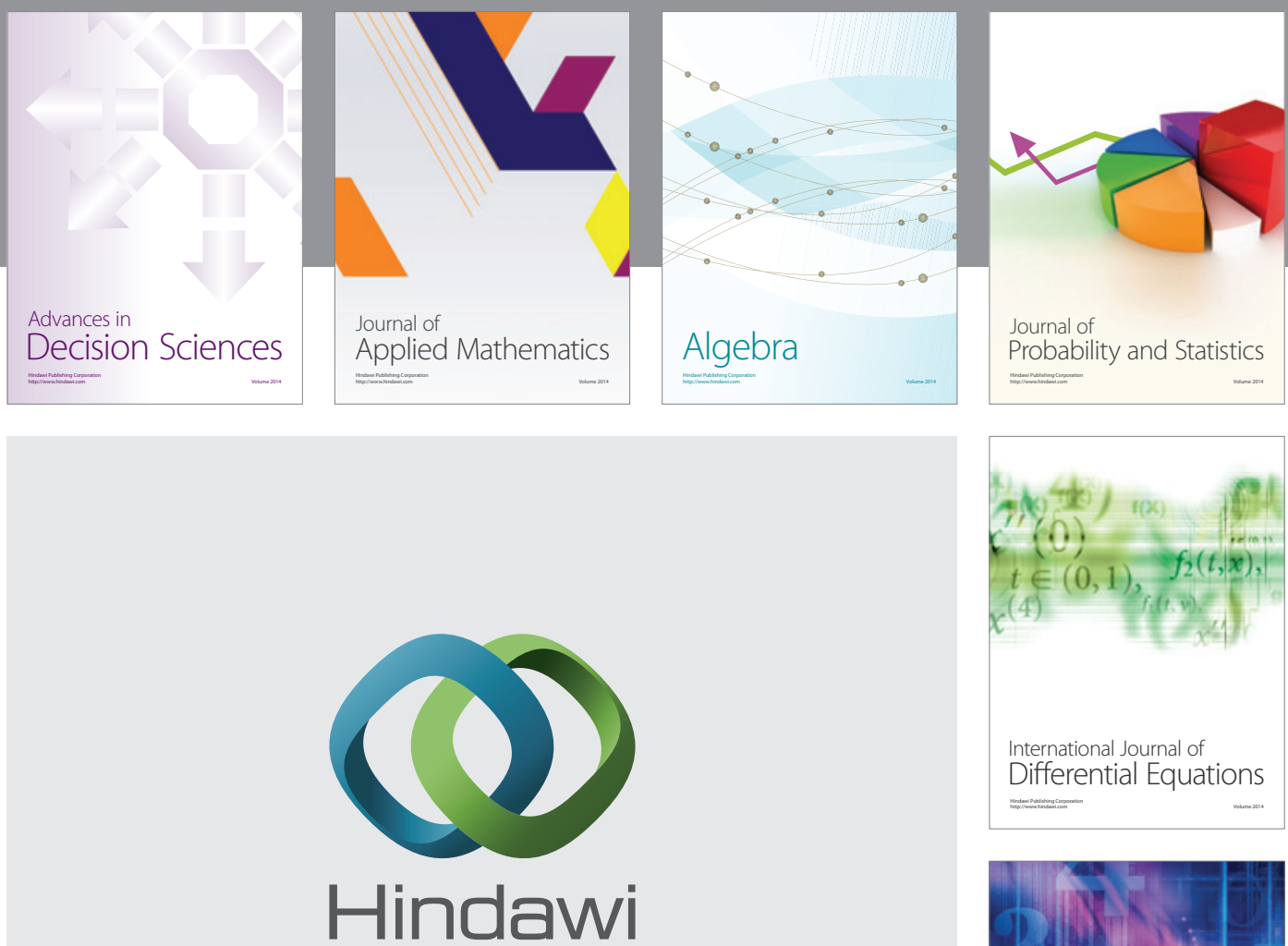

Submit your manuscripts at http://www.hindawi.com
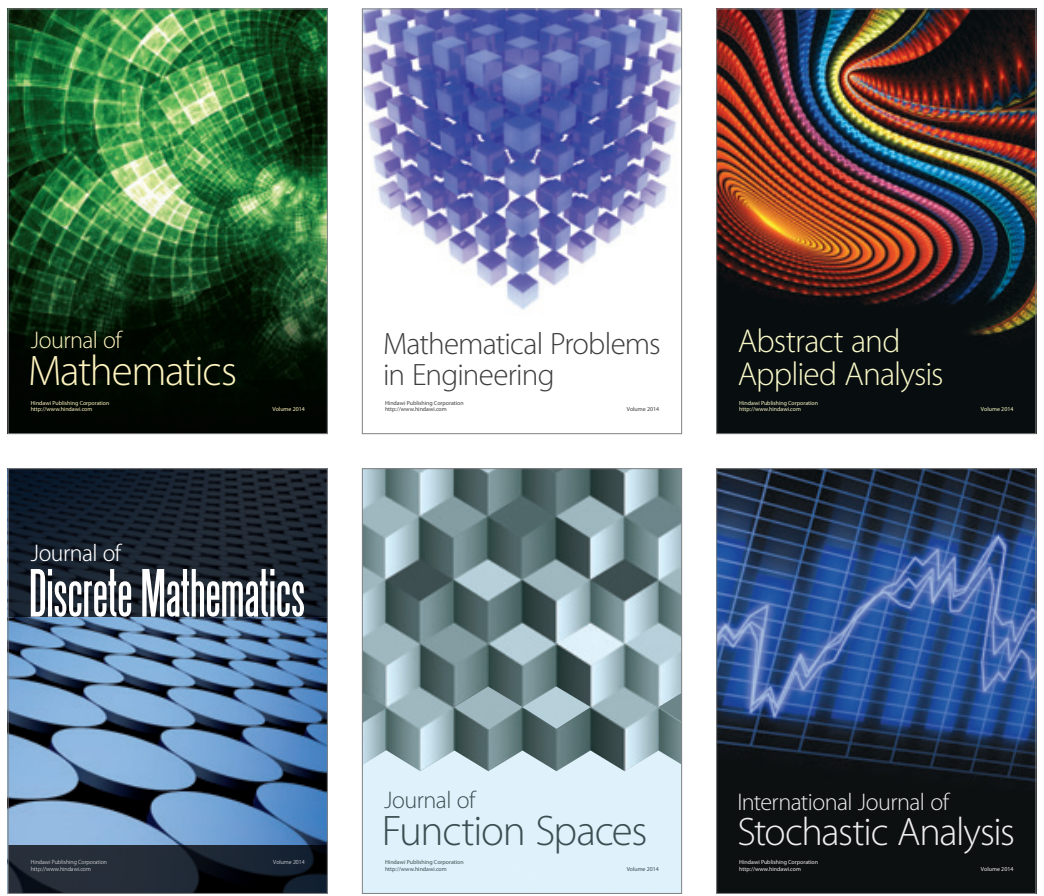

Journal of

Function Spaces

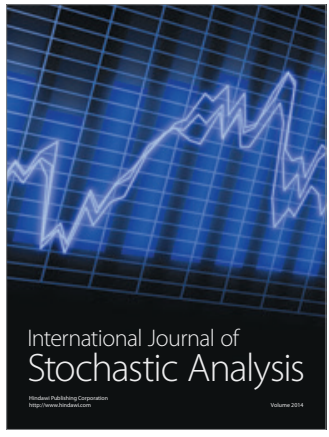

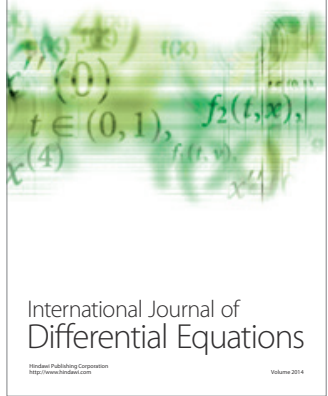
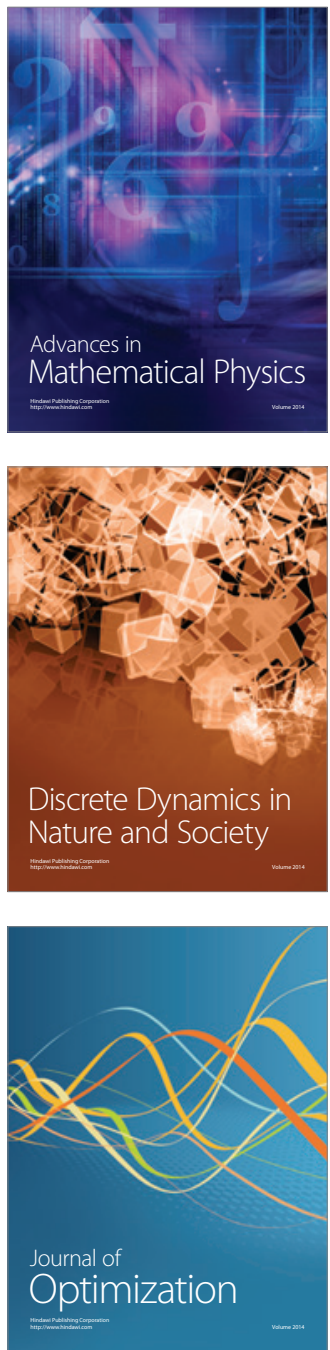